\title{
A generalization of Hawking's black hole topology theorem to higher dimensions
}

\author{
Gregory J. Galloway \\ Department of Mathematics \\ University of Miami \\ Richard Schoen \\ Department of Mathematics \\ Stanford University
}

\begin{abstract}
Hawking's theorem on the topology of black holes asserts that cross sections of the event horizon in 4-dimensional asymptotically flat stationary black hole spacetimes obeying the dominant energy condition are topologically 2spheres. This conclusion extends to outer apparent horizons in spacetimes that are not necessarily stationary. In this paper we obtain a natural generalization of Hawking's results to higher dimensions by showing that cross sections of the event horizon (in the stationary case) and outer apparent horizons (in the general case) are of positive Yamabe type, i.e., admit metrics of positive scalar curvature. This implies many well-known restrictions on the topology, and is consistent with recent examples of five dimensional stationary black hole spacetimes with horizon topology $S^{2} \times S^{1}$. The proof is inspired by previous work of Schoen and Yau on the existence of solutions to the Jang equation (but does not make direct use of that equation).
\end{abstract}




\section{Introduction}

A basic result in the theory of black holes is Hawking's theorem [11, 13] on the topology of black holes, which asserts that cross sections of the event horizon in 4-dimensional asymptotically flat stationary black hole spacetimes obeying the dominant energy condition are spherical (i.e., topologically $S^{2}$ ). The proof is a beautiful variational argument, showing that if a cross section has genus $\geq 1$ then it can be deformed along a null hypersurface to an outer trapped surface outside of the event horizon, which is forbidden by standard results on black holes [13. ${ }^{1}$ In [12, Hawking showed that his black hole topology result extends, by a similar argument, to outer apparent horizons in black hole spacetimes that are not necessarily stationary. (A related result had been shown by Gibbons $[8$ in the time-symmetric case.) Since Hawking's arguments rely on the Gauss-Bonnet theorem, these results do not directly extend to higher dimensions.

Given the current interest in higher dimensional black holes, it is of interest to determine which properties of black holes in four spacetime dimensions extend to higher dimensions. In this note we obtain a natural generalization of Hawking's theorem on the topology of black holes to higher dimensions. The conclusion in higher dimensions is not that the horizon topology is spherical; that would be too strong, as evidenced by the striking example of Emparan and Reall [7] of a stationary vacuum black hole spacetime in five dimensions with horizon topology $S^{2} \times S^{1}$. The natural conclusion in higher dimensions is that cross sections of the event horizon (in the stationary case), and outer apparent horizons (in the general case) are of positive Yamabe type, i.e. admit metrics of positive scalar curvature. As noted in [6], in the time symmetric case this follows from the minimal surface methodology of Schoen and Yau [18] in their treatment of manifolds of positive scalar curvature. The main point of the present paper is to show that this conclusion remains valid without any condition on the extrinsic curvature of space. That such a result might be expected to hold is suggested by work in [19, Section 4], which implies that the apparent horizons corresponding to the blow-up of solutions of the Jang equation, as described in [19], are of positive Yamabe type. We emphasize, however, that we do not need to make use of the Jang equation here. ${ }^{2}$

Much is now known about the topological obstructions to the existence of metrics of positive scalar curvature in higher dimensions. While the first major result along these lines is the famous theorem of Lichnerowicz [16] concerning the vanishing of the $\hat{A}$ genus, a key advance in our understanding was made in the late 70's and early 80's by Schoen and Yau [17, 18, and Gromov and Lawson [9, 10]. A brief review of these

\footnotetext{
${ }^{1}$ Actually the torus $T^{2}$ arises as a borderline case in Hawking's argument, but can occur only under special circumstances.

${ }^{2}$ In any case, the parametric estimates of [19] which are used to construct solutions of the Jang equation asymptotic to vertical cylinders over apparent horizons are generally true only in low dimensions.
} 
results, relevant to the topology of black holes, was considered in [6]. We shall recall the situation in five spacetime dimensions in the next section, after the statement of our main result.

\section{The main result}

Let $V^{n}$ be an $n$-dimensional, $n \geq 3$, spacelike hypersurface in a spacetime $\left(M^{n+1}, g\right)$. Let $\Sigma^{n-1}$ be a closed hypersurface in $V^{n}$, and assume that $\Sigma^{n-1}$ separates $V^{n}$ into an "inside" and an "outside". Let $N$ be the outward unit normal to $\Sigma^{n-1}$ in $V^{n}$, and let $U$ be the future directed unit normal to $V^{n}$ in $M^{n+1}$. Then $K=U+N$ is an outward null normal field to $\Sigma^{n-1}$, unique up to scaling.

The null second fundamental form of $\Sigma$ with respect to $K$ is, for each $p \in \Sigma$, the bilinear form defined by,

$$
\chi: T_{p} \Sigma \times T_{p} \Sigma \rightarrow \mathbb{R}, \quad \chi(X, Y)=\left\langle\nabla_{X} K, Y\right\rangle
$$

where $\langle\rangle=$,$g and \nabla$ is the Levi-Civita connection, of $M^{n+1}$. Then the null expansion of $\Sigma$ is defined as $\theta=\operatorname{tr} \chi=h^{A B} \chi_{A B}=\operatorname{div}_{\Sigma} K$, where $h$ is the induced metric on $\Sigma$.

We shall say $\Sigma^{n-1}$ is an outer apparent horizon in $V^{n}$ provided, (i) $\Sigma$ is marginally outer trapped, i.e., $\theta=0$, and (ii) there are no outer trapped surfaces outside of $\Sigma$. The latter means that there is no $(n-1)$-surface $\Sigma^{\prime}$ contained in the region of $V^{n}$ outside of $\Sigma$ which is homologous to $\Sigma$ and which has negative expansion $\theta<0$ with respect to its outer null normal (relative to $\Sigma$ ). Heuristically, $\Sigma$ is the "outer limit" of outer trapped surfaces in $V$.

Finally, a spacetime $\left(M^{n+1}, g\right)$ satisfying the Einstein equations

$$
R_{a b}-\frac{1}{2} R g_{a b}=T_{a b}
$$

is said to obey the dominant energy condition provided the energy-momentum tensor $\mathcal{T}$ satisfies $\mathcal{T}(X, Y)=T_{a b} X^{a} Y^{b} \geq 0$ for all future pointing causal vectors $X, Y$.

We are now ready to state the main theorem.

Theorem 2.1 Let $\left(M^{n+1}, g\right), n \geq 3$, be a spacetime satisfying the dominant energy condition. If $\Sigma^{n-1}$ is an outer apparent horizon in $V^{n}$ then $\Sigma^{n-1}$ is of positive Yamabe type, unless $\Sigma^{n-1}$ is Ricci flat (flat if $n=3,4$ ) in the induced metric, and both $\chi$ and $\mathcal{T}(U, K)=T_{a b} U^{a} K^{b}$ vanish on $\Sigma$.

Thus, except under special circumstances, $\Sigma^{n-1}$ is of positive Yamabe type. As noted in the introduction, this implies various restrictions on the topology of $\Sigma$. Let us focus on the case $\operatorname{dim} M=5$, and hence $\operatorname{dim} \Sigma=3$, and assume, by taking a double cover if necessary, that $\Sigma$ is orientable. Then by well-known results of SchoenYau [18] and Gromov-Lawson [10], topologically, $\Sigma$ must be a finite connected sum 
of spherical spaces (homotopy 3-spheres, perhaps with identifications) and $S^{2} \times S^{1}$ 's. Indeed, by the prime decomposition theorem, $\Sigma$ can be expressed as a connected sum of spherical spaces, $S^{2} \times S^{1}$ 's, and $K(\pi, 1)$ manifolds (manifolds whose universal covers are contractible). But as $\Sigma$ admits a metric of positive scalar curvature, it cannot have any $K(\pi, 1)$ 's in its prime decomposition. Thus, the basic horizon topologies in $\operatorname{dim} M=5$ are $S^{3}$ and $S^{2} \times S^{1}$, both of which are realized by nontrivial black hole spacetimes. Under stringent geometric assumptions on the horizon, a related conclusion is arrived at in [14].

Proof of the theorem: We consider normal variations of $\Sigma$ in $V$, i.e., variations $t \rightarrow \Sigma_{t}$ of $\Sigma=\Sigma_{0},-\epsilon<t<\epsilon$, with variation vector field $V=\left.\frac{\partial}{\partial t}\right|_{t=0}=\phi N, \phi \in C^{\infty}(\Sigma)$. Let $\theta(t)$ denote the null expansion of $\Sigma_{t}$ with respect to $K_{t}=U+N_{t}$, where $N_{t}$ is the outer unit normal field to $\Sigma_{t}$ in $V$. A computation shows [6, 3],

$$
\left.\frac{\partial \theta}{\partial t}\right|_{t=0}=-\triangle \phi+2\langle X, \nabla \phi\rangle+\left(Q+\operatorname{div} X-|X|^{2}\right) \phi,
$$

where,

$$
Q=\frac{1}{2} S-\mathcal{T}(U, K)-\frac{1}{2}|\chi|^{2},
$$

$S$ is the scalar curvature of $\Sigma, X$ is the vector field on $\Sigma$ defined by $X=\tan \left(\nabla_{N} U\right)$, and $\langle$,$\rangle now denotes the induced metric h$ on $\Sigma$.

Introducing as in [3] the operator $L=-\triangle+\langle X, \nabla()\rangle+\left(Q+\operatorname{div} X-|X|^{2}\right)$, Equation (2.3) may be expressed as,

$$
\left.\frac{\partial \theta}{\partial t}\right|_{t=0}=L(\phi)
$$

$L$ is the stability operator associated with variations in the null expansion $\theta$. In the time symmetric case the vector field $X$ vanishes, and $L$ reduces to the classical stability operator of minimal surface theory, as expected [6].

As discussed in [3], although $L$ is not in general self adjoint, its principal eigenvalue $\lambda_{1}$ is real, and one can choose a principal eigenfunction $\phi$ which is strictly positive, $\phi>0$. Using the eigenfunction $\phi$ to define our variation, we have from (2.5),

$$
\left.\frac{\partial \theta}{\partial t}\right|_{t=0}=\lambda_{1} \phi
$$

The eigenvalue $\lambda_{1}$ cannot be negative, for otherwise (2.6) would imply that $\frac{\partial \theta}{\partial t}<0$ on $\Sigma$. Since $\theta=0$ on $\Sigma$, this would mean that for $t>0$ sufficiently small, $\Sigma_{t}$ would be outer trapped, contrary to our assumptions.

Hence, $\lambda_{1} \geq 0$, and we conclude for the variation determined by the positive eigenfunction $\phi$ that $\left.\frac{\partial \theta}{\partial t}\right|_{t=0} \geq 0$. By completing the square on the right hand side of Equation (2.3), this implies that the following inequality holds,

$$
-\triangle \phi+(Q+\operatorname{div} X) \phi+\phi|\nabla \ln \phi|^{2}-\phi|X-\nabla \ln \phi|^{2} \geq 0 .
$$


Setting $u=\ln \phi$, we obtain,

$$
-\triangle u+Q+\operatorname{div} X-|X-\nabla u|^{2} \geq 0 .
$$

As a side remark, note that substituting the expression for $Q$ into (2.8) and integrating gives that the total scalar curvature of $\Sigma$ is nonnegative, and in fact is positive, except under special circumstances. In four spacetime dimensions one may then apply the Gauss-Bonnet theorem to recover Hawking's theorem; in fact this is essentially Hawking's original argument. However, in higher dimensions the positivity of the total scalar curvature, in and of itself, does not provide any topological information.

To proceed with the higher dimensional case, we first absorb the Laplacian term $\Delta u=\operatorname{div}(\nabla u)$ in (2.8) into the divergence term to obtain,

$$
Q+\operatorname{div}(X-\nabla u)-|X-\nabla u|^{2} \geq 0 .
$$

Setting $Y=X-\nabla u$, we arrive at the inequality,

$$
-Q+|Y|^{2} \leq \operatorname{div} Y
$$

Given any $\psi \in C^{\infty}(\Sigma)$, we multiply through by $\psi^{2}$ and derive,

$$
\begin{aligned}
-\psi^{2} Q+\psi^{2}|Y|^{2} & \leq \psi^{2} \operatorname{div} Y \\
& =\operatorname{div}\left(\psi^{2} Y\right)-2 \psi\langle\nabla \psi, Y\rangle \\
& \leq \operatorname{div}\left(\psi^{2} Y\right)+2|\psi||\nabla \psi||Y| \\
& \leq \operatorname{div}\left(\psi^{2} Y\right)+|\nabla \psi|^{2}+\psi^{2}|Y|^{2} .
\end{aligned}
$$

Integrating the above inequality yields,

$$
\int_{\Sigma}|\nabla \psi|^{2}+Q \psi^{2} \geq 0 \quad \text { for all } \psi \in C^{\infty}(\Sigma)
$$

where $Q$ is given in (2.4).

At this point rather standard arguments become applicable [19, 6]. Consider the eigenvalue problem,

$$
-\triangle \psi+Q \psi=\mu \psi
$$

Inequality (2.12) implies that the first eigenvalue $\mu_{1}$ of (2.13) is nonnegative, $\mu_{1} \geq 0$. Let $f \in C^{\infty}(\Sigma)$ be an associated eigenfunction; $f$ can be chosen to be strictly positive.

Now consider $\Sigma$ in the conformally related metric $\tilde{h}=f^{2 / n-2} h$. The scalar curvature $\tilde{S}$ of $\Sigma$ in the metric $\tilde{h}$ is given by,

$$
\begin{aligned}
\tilde{S} & =f^{-n /(n-2)}\left(-2 \Delta f+S f+\frac{n-1}{n-2} \frac{|\nabla f|^{2}}{f}\right) \\
& =f^{-2 /(n-2)}\left(2 \mu_{1}+2 \mathcal{T}(U, K)+|\chi|^{2}+\frac{n-1}{n-2} \frac{|\nabla f|^{2}}{f^{2}}\right),
\end{aligned}
$$


where, for the second equation, we have used (2.13), with $\psi=f$, and (2.4).

Since, by the dominant energy condition, $\mathcal{T}(U, K) \geq 0$, Equation (2.14) implies that $\tilde{S} \geq 0$. If $\tilde{S}>0$ at some point, then by well known results [15] one can conformally change $\tilde{h}$ to a metric of strictly positive scalar curvature, and the theorem follows. If $\tilde{S}$ vanishes identically then, by Equation (2.14) $, \mu_{1}=0, \mathcal{T}(U, K) \equiv 0$, $\chi \equiv 0$ and $f$ is constant. Equation (2.13), with $\psi=f$ and Equation (2.4) then imply that $S \equiv 0$. By a result of Bourguinon (see [15]), it follows that $\Sigma$ carries a metric of positive scalar curvature unless it is Ricci flat. The theorem now follows.

\section{Concluding Remarks.}

1. Let $\Sigma^{n-1}$ be a closed 2-sided hypersurface in the spacelike hypersurface $V^{n} \subset M^{n+1}$. Then there exists a neighborhood $W$ of $\Sigma^{n-1}$ in $V^{n}$ such that $\Sigma$ separates $W$ into an "inside" and an "outside". Suppose $\Sigma$ is marginally outer trapped, i.e., $\theta=0$ with respect to the outer null normal to $\Sigma$. Following the terminology introduced in [3], we say that $\Sigma$ is stably outermost (respectively, strictly stably outermost) provided the principal eigenvalue $\lambda_{1}$ of the stability operator $L$ introduced in 2.5 satisfies $\lambda_{1} \geq 0$ (resp., $\lambda_{1}>0$ ). It is clear from the proof that the conclusion of Theorem 2.1 remains valid for marginally outer trapped surfaces $\Sigma$ that are stably outermost. Moreover the conclusion that $\Sigma$ is positive Yamabe holds without any caveat if $\Sigma$ is strictly stably outermost. To see this, note that Equation (2.6) then implies that there exists

$\epsilon>0$ such that $\left.\frac{\partial \theta}{\partial t}\right|_{t=0} \geq \epsilon$. Tracing through the proof using this inequality shows that (2.12) holds with $Q$ replaced by $Q-\epsilon$. Then the parenthetical expression in Equation (2.14) will include a $+\epsilon$ term, and so $\tilde{S}$ will be strictly positive.

2. Theorem 2.1 applies, in particular, to the marginally trapped surfaces $S_{R}$ of a dynamical horizon $\mathcal{H}$ (see 2] for definitions). Indeed, by the maximum principle for marginally trapped surfaces [1], there can be no outer trapped surfaces in $\mathcal{H}$ outside of any $S_{R}$. Alternatively, it is easily checked that each $S_{R}$ is stably outermost in the sense described in the previous paragraph.

3. As discussed in [6], the exceptional case in Theorem 2.1 can in effect be eliminated in the time symmetric case. In this case $V^{n}$ becomes a manifold of nonnegative scalar curvature, and $\Sigma^{n-1}$ is minimal. By the results in [5, 4, if $\Sigma$ is locally outer area minimizing and does not carry a metric of positive scalar curvature then an outer neighborhood of $\Sigma$ in $V$ splits isometrically as a product $[0, \epsilon) \times \Sigma$. In physical terms, this means that there would be marginally outer trapped surfaces outside of $\Sigma$, which, by a slight strengthening of our definition of 'outer apparent horizon', could not occur. (In fact, marginally outer trapped surfaces cannot occur outside the event horizon.) Under mild physical assumptions, but with $\operatorname{dim} M \leq 8$, one can show that $\Sigma$ is locally outer area minimizing; see [6, Theorem 3] for further discussion. Finally, in the asymptotically flat, but not necessarily time symmetric case, it is possible to perturb the initial data to make the dominant energy inequality strict, see [19, p. 240]. Hence, the exceptional case is unstable in this sense. 
Acknowledgements. This work was supported in part by NSF grants DMS-0405906 (GJG) and DMS-0104163 (RS). The work was initiated at the Isaac Newton Institute in Cambridge, England during the Fall 2005 Program on Global Problems in Mathematical Relativity, organized by P. Chruściel, H. Friedrich and P. Tod. The authors would like to thank the Newton Institute for its support.

\section{References}

[1] A. Ashtekar and G. J. Galloway, Uniqueness theorems for dynamical horizons, to appear in Adv. Theor. Math. Phys., gr-qc/0503109.

[2] A. Ashtekar and B. Krishnan, Dynamical horizons and their properties, Phys. Rev. D 68 (2003) 261101.

[3] L. Andersson, M. Mars, and W. Simon, Local existence of dynamical and trapping horizons, Phys. Rev. Lett. 95 (2005) 111102.

[4] M. Cai, Volume minimizing hypersurfaces in manifolds of nonnegative scalar curvature, in: Minimal Surfaces, Geometric Analysis, and Symplectic Geometry, Advanced Studies in Pure Mathematics, eds. K. Fukaya, S. Nishikawa and J. Spruck, 34 (2002) 1-7.

[5] M. Cai and G. J. Galloway, Rigidity of area minimzing tori in 3-manifolds of nonnegative scalar curvature, Commun. Anal. Geom. 8 (2000) 565-573.

[6] - On the topology and area of higher dimensional black holes, Class. Quant. Grav. 18 (2001) 2707-2718.

[7] R. Emparan and H. S. Reall, A rotating black ring in five dimensions, Phys. Rev. Lett. 88 (2002) 101101

[8] G. W. Gibbons, The time symmetric initial value problem for black holes, Commun. Math. Phys. 27 (1972) 87-102.

[9] M. Gromov and B. Lawson, Spin and scalar curvature in the presence of the fundamental group, Ann. of Math. 111 (1980) 209-230.

[10] _ Positive scalar curvature and the Dirac operator on complete Riemannian manifolds, Publ. Math. IHES 58 (1983) 83-196.

[11] S. W. Hawking, Black holes in general relativity, Commun. Math. Phys. 25 (1972) 152-166.

[12] — The event horizon, in 'Black Holes, Les Houches lectures' (1972), edited by C. DeWitt and B. S. DeWitt (North Holland, Amsterdam, 1972). 
[13] S. W. Hawking and G. F. R. Ellis, The large scale structure of space-time, Cambridge University Press, Cambridge, 1973.

[14] On the topology of black hole event horizons in higher dimensions, C. Helfgott, Y. Oz, and Y. Yanay, hep-th/0509013.

[15] J. Kazdan and F. Warner, Prescribing curvatures, Proc. Symp. in Pure Math. 27 (1975) 309-319.

[16] A. Lichnerowicz, Spineurs harmoniques, Cr. Acd. Sci. Paris, Sér. A-B 257 (1963) $7-9$.

[17] R. Schoen and S. T. Yau, Existence of incompressible minimal surfaces and the topology of three dimensional manifolds of non-negative scalar curvature, Ann. of Math. 110 (1979) 127-142.

[18] _ On the structure of manifolds with positive scalar curvature, Manuscripta Math. 28 (1979) 159-183.

[19] _ Proof of the positive of mass theorem. II, Commun. Math. Phys., 79 (1981) 231-260. 